\title{
Colossal electroresistance without colossal magnetoresistance in $\mathrm{La}_{0.9} \mathrm{Sr}_{0.1} \mathrm{MnO}_{3}$
}

\author{
N. Biškup, ${ }^{\text {a) }}$ A. de Andrés, N. M. Nemes, and M. García-Hernandez \\ Instituto de Ciencia de Materiales, CSIC, Cantoblanco, 28049 Madrid, Spain \\ K. V. Glazyrin and Y. M. Mukovskii \\ Moscow State Steel and Alloys Institute, Leninskii prosp. 4, Moscow 119049, Russia
}

(Received 29 March 2007; accepted 9 May 2007; published online 31 May 2007)

\begin{abstract}
The authors report on colossal electroresistance (ER) in the ferromagnetic insulator manganite $\mathrm{La}_{0.9} \mathrm{Sr}_{0.1} \mathrm{MnO}_{3}$. The single crystal samples exhibit a transition into a low resistive state above a certain threshold current. Pulsed measurements demonstrate that this transition is not a consequence of heating. ER behaves similarly to magnetoresistance (MR) above the orbital order temperature $T_{\mathrm{OO}}$. Below $T_{\mathrm{OO}}$ the MR is only $20 \%$ while ER enhances the conductivity by several orders of magnitude. Magnetic field and electric current have opposite effects on the conductivity, therefore, it seems that only the injected carriers are able to modify the ordering of $e_{g}$ Mn orbitals. (c) 2007 American Institute of Physics. [DOI: 10.1063/1.2745220]
\end{abstract}

Materials and composites based on mixed manganese oxides (manganites) $R_{1-x} A_{x} \mathrm{MnO}_{3}(R=\mathrm{La}, \mathrm{Pr} ; A=\mathrm{Ca}, \mathrm{Sr})$ are prospect candidates for electronic and/or magnetic devices. Their potential for application is based on coupling of charge and spin orders of freedom. The well studied phenomenon in manganites is magnetoresistance (MR), the magnitude of which ranges from "giant" $10 \%-20 \%$ to colossal $10^{8} \%$ in charge ordered $\mathrm{Pr}_{1-x} \mathrm{Ca}_{x} \mathrm{MnO}_{3}, x=0.3-0.5{ }^{1}$ Recently, a more debated phenomenon is electroresistance (ER), i.e., nonlinear current-voltage $(I-V)$ characteristics. Similar to MR, the magnitude of ER varies in different compounds, reaching equally colossal values in $\operatorname{Pr}_{0.7} \mathrm{Ca}_{0.3} \mathrm{MnO}_{3}{ }^{2}{ }^{2} \mathrm{ER}$ and MR are coupled, i.e., the voltage/current necessary to trigger insulator-metal transition decreases with magnetic field. Since this initial report in 1997, a significant number of publications report nonlinear $I-V$ effects in manganites. Initially, investigations were limited to materials with charge order (CO) ground state. Different interpretations for these nonlinearities included depinning of $\mathrm{CO}$ state, ${ }^{3}$ depinning of charge density waves, ${ }^{4}$ or change in the orientation of orbital ordering (OO). ${ }^{5,6}$ Indeed, nonlinear effects were subsequently found in materials without $\mathrm{CO},{ }^{7,8}$ undermining the interpretation of "melting" of CO/OO states.

Recent reports tend to interpret the nonlinear $I-V$ characteristics in manganites as the consequence of Joule heating due to large currents. ${ }^{9-11}$ In the case of resistive curves with a maximum in $R(T)$, current localization on conductive paths and self-heating was suggested to explain the concomitant increase and decrease of resistance with increasing current. $^{12,13}$ However, abrupt switches between high and low resistive states are also found in systems where heating cannot explain the increase of resistance with increasing current. $^{14}$

To elucidate the relationship of $\mathrm{CO} / \mathrm{OO}$ order, magnetism, heating, and $I-V$ nonlinearities in manganites, we have studied $\mathrm{La}_{0.9} \mathrm{Sr}_{0.1} \mathrm{MnO}_{3}$, a system that is a ferromagnetic insulator with charge/orbital ordering. ${ }^{15}$ The parent compound $\mathrm{LaMnO}_{3}$ is a paramagnetic insulator that at $T<140 \mathrm{~K}$ orders

\footnotetext{
${ }^{a)}$ Electronic mail: biskup@icmm.csic.es
}

antiferromagnetically. By hole doping (by divalent ions such as $\mathrm{Sr}^{2+}$ ) it is possible to change the ground state from insulating to metallic. For $\mathrm{La}_{1-x} \mathrm{Sr}_{x} \mathrm{MnO}_{3}$ this happens for $x>0.175,{ }^{15}$ with the ground state being a ferromagnetic metal. On the contrary, the title compound $(x=0.1)$, despite ordering ferromagnetically at $T_{c} \approx 150 \mathrm{~K}$, remains insulating in the whole temperature range. The $x-T$ phase diagram of low-doped $(x<0.175) \mathrm{La}_{1-x} \mathrm{Sr}_{x} \mathrm{MnO}_{3}$ exhibits several structural and magnetic transitions, ${ }^{16}$ with a much debated ground state. ${ }^{17-20}$ Recent reports seem to agree that OO is responsible for the insulating behavior of $\mathrm{La}_{0.9} \mathrm{Sr}_{0.1} \mathrm{MnO}_{3}$ despite its ferromagnetism. ${ }^{21,22}$ The character of the different phase transitions in manganites is an important point of debate. First order transitions are usually related to colossal values of MR and to metal-insulator transitions strongly coupled to structural rearrangements where Jahn-Teller distortions and/or orbital order are present. In the results presented here, all these ingredients are there but the transition is promoted by carrier injection, leading to huge ER but not by an external magnetic field.

Single crystals of $\mathrm{La}_{0.9} \mathrm{Sr}_{0.1} \mathrm{MnO}_{3}$ were prepared by the floating zone technique. ${ }^{23}$ Transport measurements were performed with several different models of current sources and voltmeters in order to eliminate the possibility of experimental artifacts. At low temperatures where the resistance diverges $(T<70 \mathrm{~K}) I$ - $V$ curves were taken in constant voltage mode, i.e., measuring current. In the range $70<T<300 \mathrm{~K}$, $\mathrm{dc}$ measurements (in constant current mode and in agreement with the constant voltage mode near $70 \mathrm{~K}$ ) were accompanied by pulsed measurements (pulse width of $0.5 \mathrm{~ms}$ ). Magnetization measurements and resistivity in magnetic field are carried out in Quantum Design magnetic property measurement system (magnetometer) and physical property measurement system, respectively.

Figure 1 shows a typical $I-V$ curve taken at $T=77 \mathrm{~K}$. The sample is immersed directly into liquid nitrogen in order to minimize Joule heating. Current cycle was $I=0 \rightarrow I_{\max } \rightarrow-I_{\max } \rightarrow 0$ (black points). The $I$ - $V$ curve departs from linear Ohmic behavior for rather low currents, and at the threshold current $I_{t}$ exhibits a sharp transition from high resistive (HR) to low resistive (LR) state. With current de- 


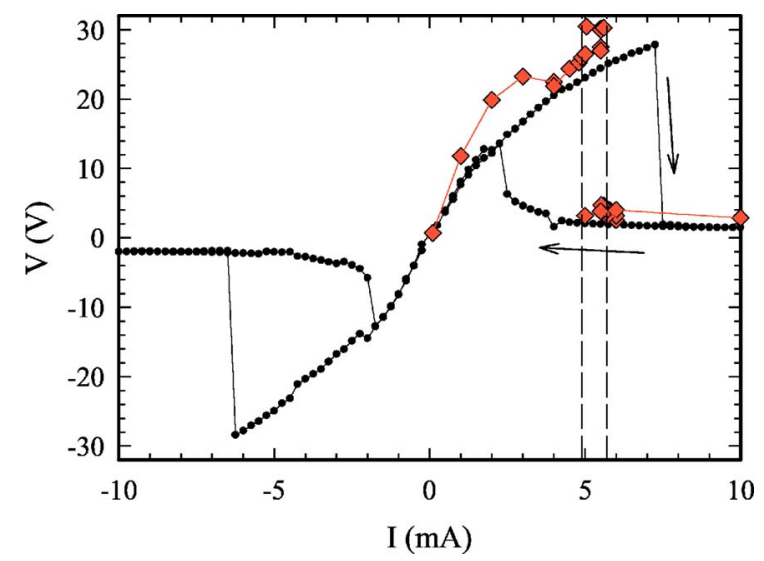

FIG. 1. (Color online) $I-V$ characteristic of $\mathrm{La}_{0.9} \mathrm{Sr}_{0.1} \mathrm{MnO}_{3}$ at $T=77 \mathrm{~K}$. Black points: $I=0 \rightarrow I_{\max } \rightarrow-I_{\max } \rightarrow 0$ current sweep. Arrows denote the direction of current sweep. Red diamonds: voltage for time separated $[\Delta(p$ $-p)=2 \mathrm{~min}]$ current pulses. Lines are guides to the eyes for the HR and LR regions.

creasing back to zero, the sample stays in the LR state down to smaller values of current, resulting in a large hysteresis. This suggests that current history influences the resistive state of $\mathrm{La}_{0.9} \mathrm{Sr}_{0.1} \mathrm{MnO}_{3}$. In addition, Fig. 1 also shows an $I-V$ curve for separate pulses of dc current (red diamonds). These data are taken instantaneously after current switch on $(\Delta t \leqslant 10 \mathrm{~ms}$ and $2 \mathrm{~min}$ passed between each current switch on). Similar to the uninterrupted current cycle, this $I-V$ curve shows a clear distinction between the high resistive $(I<4.9 \mathrm{~mA})$ and low resistive $(I>5.7 \mathrm{~mA})$ regimes. Between these two currents, there is a region (dashed lines) where the sample can be either in the high or low resistive state. This narrow region is always situated exactly at the center of hysteresis of the continuous current cycle, indicating that first order $\mathrm{HR} \rightarrow \mathrm{LR}$ transitions depend not only on electric current history (sweeps) but also on charge/spin/ orbital order before each current pulse. Note also that the scattering of voltage in the LR state (diamonds, $I>5.7 \mathrm{~mA}$ ) reaches up to $50 \%$. Therefore, the trigger for $\mathrm{HR} \rightarrow \mathrm{LR}$ transitions seems to be current rather than voltage. This is in accordance with the different sets of our $I-V$ measurements (not presented here) and in contrast with nonlinear conduction in charge/spin density waves $(\mathrm{C} / \mathrm{S}) \mathrm{DW}$. The conduction of $(\mathrm{C} / \mathrm{S}) \mathrm{DW}$ is triggered by an electric field (voltage) which is determined by its pinning mechanism. ${ }^{24}$ Thus, in spite of $\mathrm{CO} / \mathrm{OO}$ in our material, density wave conduction does not seem to be responsible for nonlinear conduction in $\mathrm{La}_{0.9} \mathrm{Sr}_{0.1} \mathrm{MnO}_{3}$. Our second important finding is that the absolute values of $I_{t}$ in continuous current cycles can scatter by as much as a factor of 2 . In two additional immersions into liquid nitrogen, the same sample showed identical $I$ - $V$ curves below $I_{t}$ but different threshold currents, $I_{t}=11 \mathrm{~mA}$ and $I_{t}=12 \mathrm{~mA}$. Analyzing dc and pulsed results obtained by dipping the sample in a liquid nitrogen bath we can conclude that the HR $\rightarrow$ LR transition is not caused by Joule heating since the incoming power $P=I V$ is found to be unconnected to the values of the threshold current. The observed differences in HR $\rightarrow$ LR transitions, as well as the large hysteresis (Fig. 1), are very probably related, on one hand, to the cooling process and, on the other, to the character of the current induced transition. From 300 down to $77 \mathrm{~K}$ the sample suffers several transitions, at least one magnetic transition around $145 \mathrm{~K}$ and, at $100 \mathrm{~K}$, a first order transition to an Downloaded 12 Mar 2010 to 161.111 .180 .191 . Redistribution subje
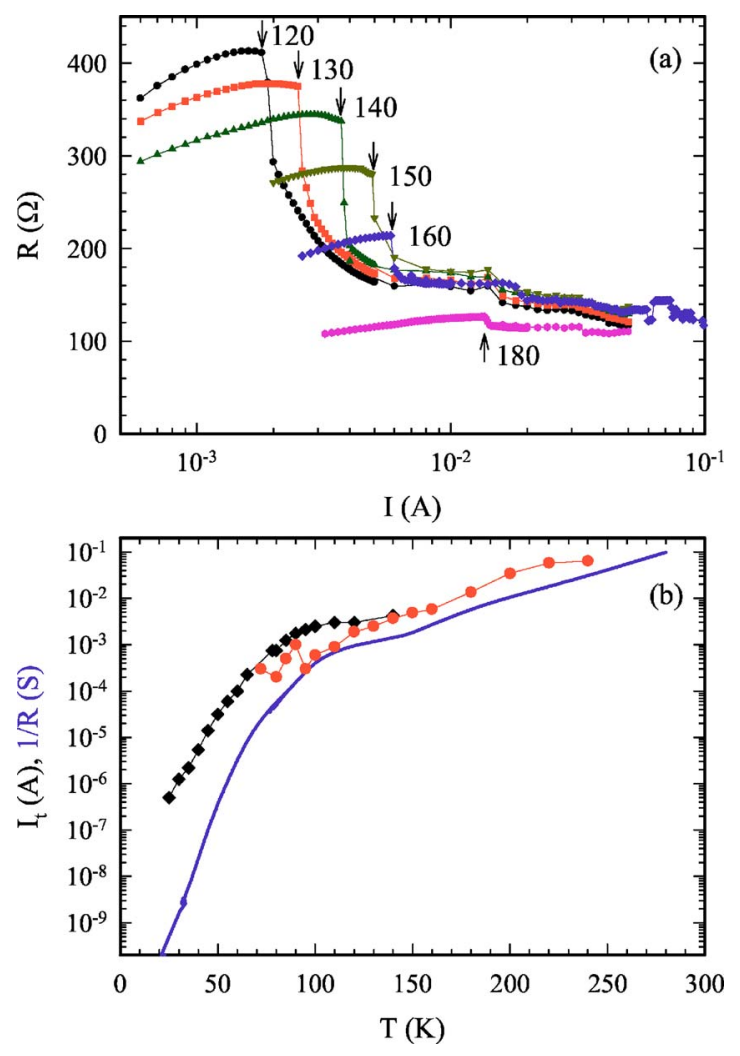

FIG. 2. (Color online) (a) Resistance $R$ measured with current pulses $(\Delta t$ $=0.5 \mathrm{~ms}$ ) as a function of current at temperatures given in the figure. Arrows indicate $\mathrm{HR} \rightarrow \mathrm{LR}$ transition. (b) Threshold current $\left(I_{t}\right)$ vs temperature. Red points and black diamonds stand for pulsed and dc measurements, respectively. Blue line shows Ohmic conductance $Y=1 / R$.

orbital ordered phase. The immersion into liquid nitrogen probably quenches the disorder of ferromagnetic/OO domains which results in different values of $I_{t}$. It is observed that slower cooling leads to higher $I_{t}$.

In order to follow the temperature behavior of $\mathrm{HR} \rightarrow$ LR transitions we have performed pulsed measurements up to room temperature. Figure 2(a) shows the dependence of the resistance $R=V I$ measured in pulses at temperatures from 120 to $180 \mathrm{~K}$. HR $\rightarrow$ LR transitions at $I=I_{t}$ are indicated by arrows. The amplitude of these discontinuous transitions drastically diminishes above the ferromagnetic order temperature $\left(T_{C}=145 \mathrm{~K}\right)$, emphasizing their relation to the ferromagnetic ordering. Figure 2(b) shows the behavior of the threshold current $I_{t}$ versus temperature. Two sets of measurements are presented: at high temperatures, the threshold current is measured in pulse technique (red points) and at low temperatures in de technique (black diamonds). The blue line corresponds to the temperature behavior of "Ohmic" conductance $Y=1 / R$, i.e., for $I \ll I_{t}$. $I_{t}$ follows Ohmic conductivity, reducing its magnitude by several orders of magnitude at low temperatures, which agrees with an electric current rather than voltage as the trigger to $\mathrm{HR} \rightarrow$ LR transitions.

Figure 3 summarizes nonlinear effects in $\mathrm{La}_{0.9} \mathrm{Sr}_{0.1} \mathrm{MnO}_{3}$ (both MR and ER) and compares them with the magnetic behavior. The magnetic susceptibility measured at 1000 Oe after zero field cooling [Fig. 3(a)] and its derivative show the bulk ferromagnetic transition, (at $T=145 \mathrm{~K}$ ) and the orbital ordering (at $T \approx 100 \mathrm{~K}$ ), which is accompanied by a magnetic rearrangement of the spins. In Fig. 3(b), continuous blue and black lines represent the temperature behavior of blue and black lines represent the temperature behavior of
to AIP license or copyright; see http://apl.aip.org/apl/copyright.jsp 


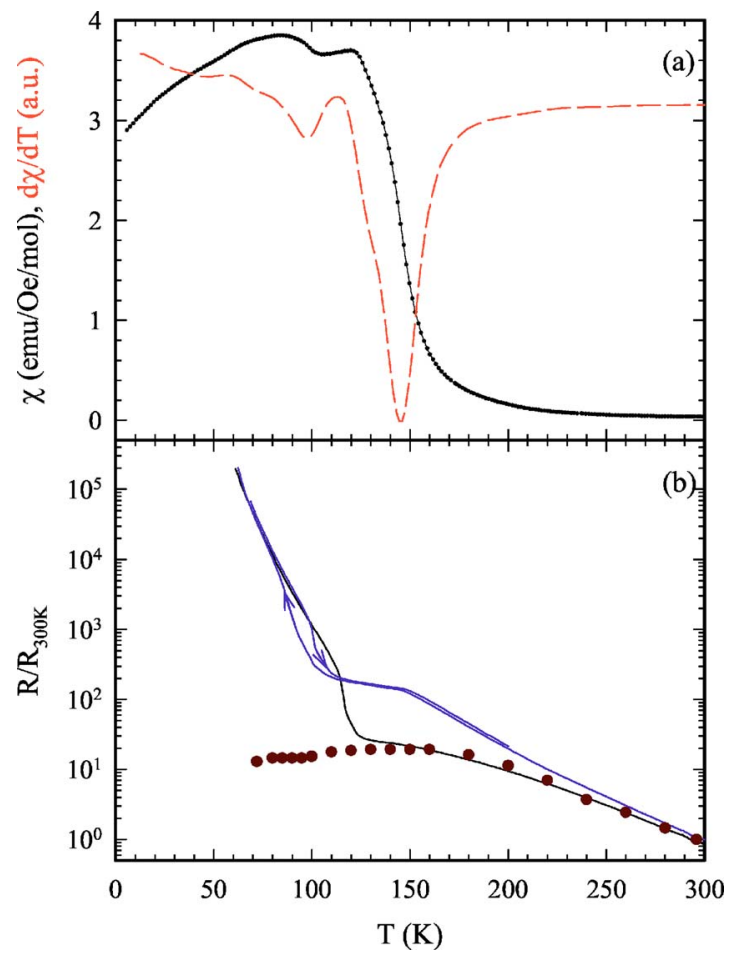

FIG. 3. (Color online) (a) Black line: magnetic susceptibility $\chi$ after zero field cooling measured in $B=0.1 \mathrm{~T}$. Red dashed line: $d \chi / d \mathrm{~T}$. (b) Temperature dependence of normalized resistance: $B=8 \mathrm{~T}$ (black line) and $B=0 \mathrm{~T}$ (blue line, with hysteresis) for $I \ll I_{t}$. Points: $B=0 \mathrm{~T}$ for $I>I_{t}$.

the HR state resistance for $B=0 \mathrm{~T}$ and $B=8 \mathrm{~T}$, respectively. Points denote LR state resistance taken from pulsed measurements (Fig. 2). The slope of the resistance at $0 \mathrm{~T}$ evidences the effects of the ferromagnetic order (strong reduction of the activation energy $E_{A}$ ) and also that of the $\mathrm{OO}$ by a sharp increase of $E_{A}$. The effect of the magnetic field on the resistance $[R(8 \mathrm{~T})]$ is what is typically seen in manganites above $T_{\mathrm{OO}}$ but produces a positive magnetoresistance around the $\mathrm{OO}$ phase transition temperature. At last, at the lower temperatures, a very small negative MR (about 20\%) is observed, quite contrary to the colossal MR observed in $\mathrm{Pr}_{1-x} \mathrm{Ca}_{x} \mathrm{MnO}_{3}$. The explanation for the MR behavior is still unclear but it seems that the magnetic field reinforces the shifting of the orbital order $T_{\mathrm{OO}}$ to higher temperatures (Fig. 3 and Ref. 20) and thus increasing the resistance. The current induced LR state resistance behaves identically to the resistance at $B=8 \mathrm{~T}$ for temperatures above $T_{\mathrm{OO}}$ which supports the equivalence of magnetic field and electrical current on transport properties in $\mathrm{La}_{0.9} \mathrm{Sr}_{0.1} \mathrm{MnO}_{3}$ at high temperatures. Both parameters lead to a large increase of the conductivity around $T_{C}$ up to one order of magnitude. The important difference arises at $T<T_{\mathrm{OO}}$. While MR is smaller than $20 \%$, carrier injection produces a reduction of the resistance of several orders of magnitude. Therefore colossal values of the ER are obtained where the resistance is almost insensitive to an external magnetic field. A possible explanation is that this colossal ER is due to the ability of the injected carriers to destroy or alter locally the orbital order while hopping from one Mn site to another. The hysteresis evidenced in Fig. 1 supports the idea that current is able to promote a different phase and that this transition may be of first order, rather than that only a local perturbation of the $\mathrm{Mn} e_{g}$ orbitals is occurring.

In summary, we have shown that a current induced electronic transition in $\mathrm{La}_{0.9} \mathrm{Sr}_{0.1} \mathrm{MnO}_{3}$ is probably of first order and leads, below $T_{\mathrm{OO}}$, to a metallic-like state, producing resistivity reduction of several orders of magnitude. This is in contrast with the effect of magnetic field that enhances the resistivity in the vicinity of $T_{\mathrm{OO}}$. ER and MR behave similarly in the ferromagnetic state above $T_{\mathrm{OO}}$.

The authors acknowledge financial support from the Ministerio de Educacion y Ciencia (MAT2005-06024C02-01 and MAT2006-01004). One of the authors (N.B.) acknowledges the Ramon and Cajal Program from the Ministerio de Educacion y Ciencia in Spain.

${ }^{1}$ Y. Tomioka, A. Asamitsu, Y. Moritomo, and Y. Tokura, J. Phys. Soc. Jpn. 64, 3626 (1995).

${ }^{2}$ A. Asamitsu, Y. Tomioka, H. Kuwahara, and Y. Tokura, Nature (London) 388, 50 (1997).

${ }^{3}$ A. Guha, A. Raychaudhuri, A. Raju, and C. N. R. Rao, Phys. Rev. B 62, 5320 (2000).

${ }^{4}$ A. Wahl, S. Mercone, A. Pautrat, M. Pollet, Ch. Simon, and D. Sedmidubsky, Phys. Rev. B 68, 094429 (2003).

${ }^{5}$ Y. Tokura and N. Nagaosa, Science 288, 462 (2000).

${ }^{6}$ K. Hatsuda, T. Kimura, and Y. Tokura, Appl. Phys. Lett. 83, 3329 (2003).

${ }^{7}$ Y. Yuzhelevski, V. Markovich, V. Dikovsky, E. Rozenberg, G. Gorodetsky, G. Jung, D. A. Shulyatev, and Ya. M. Mukovskii, Phys. Rev. B 64, $224428(2001)$.

${ }^{8}$ S. Mercone, A. Wahl, Ch.. Simon, and C. Martin, Phys. Rev. B 65, 214428 (2002).

${ }^{9}$ S. Mercone, R. Frésard, V. Caignaert, C. Martin, D. Saurel, C. Simona, G. André, P. Monod, and F. Fauth, J. Appl. Phys. 98, 023911 (2005).

${ }^{10}$ J. Sacanell, A. G. Leyva, and P. Levy, J. Appl. Phys. 98, 113708 (2005).

${ }^{11}$ Y. F. Chen, M. Ziese, and P. Esquinazi, Appl. Phys. Lett. 89, 082501 (2006).

${ }^{12}$ M. Tokunaga, Y. Tokunaga, and T. Tamegai, Phys. Rev. Lett. 93, 037203 (2004).

${ }^{13}$ A. S. Carneiro and R. F. Jardim, Phys. Rev. B 73, 012410 (2006).

${ }^{14}$ N. Biškup and A. de Andrés, Phys. Rev. B 74, 184403 (2006).

${ }^{15}$ A. Urushibara, Y. Moritomo, T. Arima, A. Asamitsu, G. Kido, and Y. Tokura, Phys. Rev. B 51, 14103 (1995).

${ }^{16}$ G.-L. Liu, J.-S. Zhou, and J. B. Goodenough, Phys. Rev. B 64, 144414 (2001).

${ }^{17}$ H. Kawano, R. Kajimoto, M. Kubota, and H. Yoshizawa, Phys. Rev. B 53, R14709 (1996).

${ }^{18}$ K. Ghosh, R. L. Greene, S. E. Lofland, S. M. Bhagat, S. G. Karabashev, D. A. Shulyatev, A. A. Arsenov, and Y. Mukovskii, Phys. Rev. B 58, 8206 (1998).

${ }^{19}$ Y. Endoh, K. Hirota, S. Ishihara, S. Okamoto, Y. Murakami, A. Nishizawa, T. Fukuda, H. Kimura, H. Nojiri, K. Kaneko, and S. Maekawa, Phys. Rev. Lett. 82, 4328 (1999).

${ }^{20}$ R. Senis, V. Laukhin, B. Martinez, J. Fontcuberta, X. Obradors, A. A. Arsenov, and Y. M. Mukovskii, Phys. Rev. B 57, 14680 (1998).

${ }^{21}$ J. Geck, P. Wochner, S. Kiele, R. Klingeler, P. Reutler, A. Revcolevschi, and B. Büchner, Phys. Rev. Lett. 95, 236401 (2005).

${ }^{22}$ G. Papavassiliou, M. Pissas, G. Diamantopoulos, M. Belesi, M. Fardis, D. Stamopoulos, A. G. Kontos, M. Hennion, J. Dolinsek, J.-Ph. Ansermet, and C. Dimitropoulos, Phys. Rev. Lett. 96, 097201 (2006).

${ }^{23}$ D. Shulyatev, S. Karabashev, A. Arsenov, Ya. Mukovskii, and S. Zverkov, J. Cryst. Growth 237-239, 810 (2002).

${ }^{24}$ G. Grüner, Rev. Mod. Phys. 66, 1 (1994). 